\title{
Metabolic Network Topology of Alzheimer's Disease and Dementia with Lewy Bodies Generated Using Fluorodeoxyglucose Positron Emission Tomography
}

\author{
Masamichi Imai $^{\mathrm{a}, \mathrm{b}, *}$, Mika Tanaka $^{\mathrm{a}}$, Muneyuki Sakata $^{\mathrm{a}}$, Kei Wagatsuma $^{\mathrm{a}}$, Tetsuro Tago ${ }^{\mathrm{a}}$, \\ Jun Toyohara ${ }^{a}$, Renpei Sengoku ${ }^{c}$, Yuji Nishina ${ }^{c}, K^{2}$ azutomi Kanemaru ${ }^{c}, K_{\text {Kenji Ishibashi }}{ }^{a}$, \\ Shigeo Murayama ${ }^{\mathrm{c}}$ and Kenji Ishii ${ }^{\mathrm{a}}$ \\ ${ }^{a}$ Team for Neuroimaging Research, Tokyo Metropolitan Institute of Gerontology, Tokyo, Japan \\ ${ }^{\mathrm{b}}$ Toranomon Hospital, Tokyo, Japan \\ ${ }^{\mathrm{c}}$ Department of Neurology, Tokyo Metropolitan Geriatric Hosptal and Institute of Gerontology, Tokyo, Japan
}

Accepted 15 October 2019

\begin{abstract}
.
Background: Alzheimer's disease (AD) and dementia with Lewy bodies (DLB) are often misdiagnosed with each other because of similar symptoms including progressive memory loss. The metabolic network topology that describes interregional metabolic connections can be generated using fluorodeoxyglucose positron emission tomography (FDG-PET) data with the graph-theoretical method. We hypothesized that different metabolic connectivity underlies the symptoms of AD patients, DLB patients, and cognitively normal (CN) individuals.

Objective: This study aimed to generate metabolic connectivity using FDG-PET data and assess the network topology to differentiate AD patients, DLB patients, and CN individuals.

Methods: This study included 45 AD patients, 18 DLB patients, and $142 \mathrm{CN}$ controls. We analyzed FDG-PET data using the graph-theoretical method and generated the network topology in AD patients, DLB patients, and CN individuals. We statistically assessed the topology with global and nodal parameters.

Results: The whole metabolic network was preserved in $\mathrm{CN}$; however, diffusely decreased connection was found in $\mathrm{AD}$ and partially but more deeply decreased connection was observed in DLB. The metabolic topology revealed that the right posterior cingulate and the left transverse temporal gyrus were significantly different between AD and DLB.

Conclusion: The present findings indicate that metabolic connectivity decreased in both AD and DLB, compared with CN. DLB was characterized restricted but deeper stereotyped network disruption compared with AD. The right posterior cingulate and the left transverse temporal gyrus are significant regions in the metabolic connectivity for differentiating AD from DLB.

Keywords: Alzheimer's disease, dementia with Lewy bodies, fluorodeoxyglucose, graph theory, network analysis, neuroimaging biomarkers, positron emission tomography
\end{abstract}

\section{INTRODUCTION}

${ }^{*}$ Correspondence to: Masamichi Imai, Tokyo Metropolitan Institute of Gerontology, 35-2 Sakae-cho, Itabashi-ku, Tokyo, 1730015, Japan. Tel.: +81 33964 3241; E-mail: imai-m@umin.ac.jp.
Alzheimer's disease (AD) and dementia with Lewy bodies (DLB) are the two primary forms of neurodegenerative dementia [1]. They are different in terms 
of clinical course, therapeutic management, and prognosis, as well as clinical diagnosis criteria; however, $\mathrm{AD}$ and DLB are often misdiagnosed with each other because both are characterized by memory loss and cognitive impairments in perception, spatial function, and constructive abilities [2-4].

Fluorodeoxyglucose positron emission tomography (FDG-PET) is commonly used for evaluating brain function. Glucose metabolism decreases in dementia including AD and DLB, compared with cognitively normal $(\mathrm{CN})$ individuals. For differentiating DLB from $\mathrm{AD}$, we can focus on hypometabolism in the visual field [5] and on relatively preserved metabolism in the posterior cingulate cortex (cingulate island sign [6]); however, we have to consider metabolic pattern overlaps in quite a few cortices in $\mathrm{AD}$ and DLB [7].

Brain networks have recently become a hot topic in the neuroscience field. Brain networks with the graph-theoretical method provide a mathematical model for quantifying structural and functional connectivity. They are studied generally using blood oxygen level-dependent (BOLD) signals that are synchronized between brain regions and functional magnetic resonance imaging (MRI) [8], which is generally conducted concomitantly in a certain task. Brain stimulation induced by the task increases regional blood supply [9]. The correlations between different activated brain regions can be visualized as simultaneous changes of blood supply (BOLD signals) with functional MRI. In addition, blood supply delivering oxygen and glucose metabolism are strongly correlated [10], and resting-state oxygen consumption and glucose utilization are also correlated [11]. Accordingly, we hypothesized that metabolic connectivity can be generated using FDGPET data, instead of functional MRI data, with the graph-theoretical method to visualize inter-regional metabolic activation.

It has been demonstrated that the functional connectivity obtained by functional MRI shows a specific pattern for AD. One of the most important connections impaired in $\mathrm{AD}$ is the default mode network (DMN) involving in episodic memory processing [12-15]. In addition, dysfunction of the DMN in mild cognitive impairment (MCI) can be used to predict the conversion from $\mathrm{MCI}$ to $\mathrm{AD}$ [16].

However, the functional connectivity in DLB obtained by functional MRI is inconsistent. For instance, Galvin et al. found significant differences in the functional connection of the precuneus to the primary visual cortex not only between DLB and
$\mathrm{CN}$, but also between DLB and AD [17]. However, Kenny et al. found no significant differences in this connection among DLB, AD, and CN [18].

In this study, we hypothesized that the metabolic connectivity obtained by FDG-PET differs among $\mathrm{AD}, \mathrm{DLB}$, and $\mathrm{CN}$ and could be used to differentiate among them. Therefore, this study aimed to assess the metabolic connectivity using FDG-PET with the graph-theoretical method to differentiate among $\mathrm{AD}$, DLB, and CN.

\section{MATERIALS AND METHODS}

\section{Participants}

This study included $45 \mathrm{AD}$ patients (30 females, $69 \pm 11$ years; 15 males, $70 \pm 10$ years), 18 DLB patients (6 females, $81 \pm 4$ years; 12 males, $75 \pm 8$ years), and $142 \mathrm{CN}$ controls (127 females, $67 \pm 5$ years; 15 males, $66 \pm 5$ years) (Table 1). Participants were recruited from those who underwent both FDGPET and MRI in our hospital from June 2000 to May 2014. Dementia was diagnosed according to the criteria of the fourth edition of the Diagnostic and Statistical Manual of Mental Disorders (DSM-IV). All patients with $\mathrm{AD}$ were classified as having probable $\mathrm{AD}$ with a high level of biomarker evidence based on "Recommendations from the National Institute on Aging-Alzheimer's Association workgroups on diagnostic guidelines for Alzheimer's disease" published in 2011 (NIA-AA 2011) [19]. Amyloid- $\beta$ biomarkers were assessed using $\left[{ }^{11} \mathrm{C}\right] \mathrm{PiB}-\mathrm{PET}$. DLB was diagnosed according to the criteria of the fourth report of the DLB Consortium [20]. CN controls had no cognitive impairment and were not taking any medications targeting at the central nervous system. Patients with notable organic brain lesions were excluded from this study. All participants provided written informed

Table 1

Characteristics of participants

\begin{tabular}{lccc}
\hline Group & $\begin{array}{c}\text { AD } \\
\text { patients }\end{array}$ & $\begin{array}{c}\text { DLB } \\
\text { patients }\end{array}$ & $\begin{array}{c}\mathrm{CN} \\
\text { individuals }\end{array}$ \\
\hline Number & 45 & 18 & 142 \\
Age $(\mathrm{y})$ & $69 \pm 11$ & $77 \pm 7$ & $67 \pm 5$ \\
Female & $67 \%(n=30)$ & $33 \%(n=6)$ & $89 \%(n=127)$ \\
MMSE & $21 \pm 7$ & $23 \pm 4$ & $29 \pm 1$ \\
\hline
\end{tabular}

All AD patients were diagnosed as having probable AD with high levels of biomarker probability according to NIA-AA 2011. All DLB patients were diagnosed using the fourth consensus report (the newest version) of the DLB Consortium. AD, Alzheimer's disease; DLB, dementia with Lewy bodies; CN, cognitive normal; MMSE, Mini-Mental State Exam. 
consent. This study was approved by the Institutional Ethics Committee of Tokyo Metropolitan Institute of Gerontology.

\section{FDG-PET imaging}

PET studies were performed using the HeadtomeV/SET 2400 W Scanner (Shimadzu, Kyoto, Japan). All patients fasted at least $5 \mathrm{~h}$ before the PET study. They were kept still on the bed and were then prepared for intravenous catheter insertion. A bolus of $150-185 \mathrm{MBq}\left[{ }^{18} \mathrm{~F}\right]-\mathrm{FDG}$ was administered in participants with a target serum glucose level of less than $140 \mathrm{mg} / \mathrm{dL}$. Attenuation was corrected by a transmission scan with ${ }^{68} \mathrm{Ga} /{ }^{68} \mathrm{Ge}$ rotating source before the emission scan. A 12-min emission scan in a 3D acquisition mode was started at $45 \mathrm{~min}$ after the injection. PET images were reconstructed using a filtered back projection method and Butterworth filter (cutoff frequency, $1.25 \mathrm{cycle} / \mathrm{cm}$; order, 2).

\section{Data processing}

All of the PET data were preprocessed using Statistical Parametric Mapping 8 (SPM8) software (Welcome Department of Imaging Neuroscience, University College, London, UK) implemented in the MatLab (Mathworks Inc, MA, USA). All of the PET images were spatially normalized to the Montreal Neurological Institute (MNI) stereotactic space and smoothed by convolution with an isotropic Gaussian filter with $16 \mathrm{~mm}$ full width at half maximum (FWHM). Standard uptake value (SUV) was calculated for each voxel, and the SUV ratio (SUVR) images were then generated compared with the mean SUV of the cerebellar cortex.

\section{Graph-theoretical descriptive measures}

To define the nodes in the graph-theoretical method, we segmented the SUVR plot to different brain regions according to an anatomical atlas of FreeSurfer version 5.1 in order to project the regions equivalent for the nodes on the spatial coordinates. The list of the regions for further analysis is described in Table 2. Subsequently, the Pearson correlation between the values of all pairs of the brain regions was calculated. The correlation denotes a connectivity matrix that represents the strength of the connection between a pair of nodes. All of the graph measures were introduced using optimized algorithms based on linear algebra to generate network construction. Graph-theoretical measures, which are used to assess the topology of the global network and its regions, were calculated in each group to adopt a method involved in Brain Connectivity Toolbox (http://www.brainconnectivity-toolbox.net/). The calculation was performed using BRAPH software based on MatLab platform [21]. The software can be used to assess the correlation in all pairs of regions according to the anatomical atlas, compute the network topology, and calculate the graph measures to describe the character of the topology. We used the BrainNet Viewer for network visualization [22].

\section{Network analysis}

To assess the global network topology in AD, DLB, and $\mathrm{CN}$, we calculated the following global parameters: 1) average strength, the average nodal strength calculated by the sum of the weights of all connections of the node; 2) average eccentricity, the average nodal maximal shortest path length between a node and any other node; 3 ) average characteristic path length, the average of the shortest path lengths between one node and all nodes; 4) average global efficiency, the average inverse shortest path length; 5) average local efficiency, the average inverse shortest path length between one node and node's neighborhood; 6) average clustering coefficient, the average nodal fraction of the degree within its neighborhood over the number of the connections that possibly exist between them; 7) transitivity, the fraction of the numbers of the triangles over the total number of the triplets; and 8) modularity, a statistic that quantifies the degree to which the topology can be divided into subnetworks. To assess the regional network, we calculated the following nodal parameters for each node: 1) nodal degree, total number of edges connected to the node; 2) nodal strength, the sum of the weights of all connections of the node; 3 ) triangles, the number of the neighboring nodes that link to each other, resulting in triangle form between a node and its neighbors; 4) nodal eccentricity, the maximal shortest path length between the node and any other node; 5) nodal path length, the shortest path lengths between the node and all the other nodes; 6) nodal clustering coefficient, fraction of the degree within its neighborhood over the number of the connections that possibly exist between them; 7) global efficiency of the node, average of the inverse shortest path length from a node to all other nodes; and 8) closeness centrality, inverse of the path length of the node. 
Table 2

Brain regions involved in the graph-theoretical method

\begin{tabular}{|c|c|c|c|c|c|c|}
\hline \multirow[t]{2}{*}{ No } & \multirow[t]{2}{*}{ Regions } & \multicolumn{3}{|c|}{$\begin{array}{l}\text { Montreal neurological } \\
\text { institute coordinates }\end{array}$} & \multirow[t]{2}{*}{$\mathrm{L} / \mathrm{R}$} & \multirow[t]{2}{*}{ label } \\
\hline & & $\mathrm{x}$ & $\mathrm{y}$ & $\mathrm{Z}$ & & \\
\hline 1 & superior frontal & -12.6 & 22.9 & 42.4 & left & $1 \mathrm{SF}$ \\
\hline 2 & frontal pole & -8.6 & 61.7 & -8.7 & left & 1FP \\
\hline 3 & rostral middle frontal & -31.3 & 41.2 & 16.5 & left & IRMF \\
\hline 4 & caudal middle frontal & -34.6 & 10.2 & 42.8 & left & $1 \mathrm{CMF}$ \\
\hline 5 & pars orbitalis & -41 & 38.8 & -11.1 & left & 1POB \\
\hline 6 & lateral orbitofrontal & -24 & 28.6 & -14.4 & left & ILOF \\
\hline 7 & pars triangularis & -42.4 & 30.6 & 2.3 & left & 1PT \\
\hline 8 & pars opercularis & -44.6 & 14.6 & 13.1 & left & 1POP \\
\hline 9 & medial orbitofrontal & -8 & 34.9 & -14.9 & left & IMOF \\
\hline 10 & rostral anterior cingulate & -6.8 & 33.9 & 1.6 & left & IRAC \\
\hline 11 & caudal anterior cingulate & -6.6 & 18 & 26.1 & left & 1CAC \\
\hline 12 & insula & -34.2 & -4.3 & 2.2 & left & IINS \\
\hline 13 & precentral & -37.8 & -10.7 & 42.1 & left & 1PRC \\
\hline 14 & postcentral & -42.3 & -23.8 & 43.6 & left & IPOC \\
\hline 15 & supramarginal & -50.4 & -38.8 & 31 & left & 1SUPRA \\
\hline 16 & superior parietal & -22.8 & -60.9 & 46.3 & left & 1SP \\
\hline 17 & inferior parietal & -40 & -66.4 & 27.3 & left & IIP \\
\hline 18 & paracentral & -10 & -28.7 & 56.1 & left & IPARAC \\
\hline 19 & posterior cingulate & -7.3 & -17.4 & 35.7 & left & 1PCG \\
\hline 20 & isthmus cingulate & -8.9 & -45.4 & 17.6 & left & IIST \\
\hline 21 & precuneus & -11.6 & -57.5 & 36.7 & left & IPREC \\
\hline 22 & cuneus & -8.7 & -79.6 & 18 & left & ICUN \\
\hline 23 & pericalcarine & -13.9 & -80.6 & 6 & left & 1PERI \\
\hline 24 & lingual & -16.5 & -66.8 & -4.3 & left & ILIN \\
\hline 25 & lateral occipital & -29.7 & -86.9 & -1 & left & $1 \mathrm{LO}$ \\
\hline 26 & transverse temporal & -44 & -24.2 & 6 & left & ITRANS \\
\hline 27 & banks superior temporal & -52.7 & -44.5 & 4.6 & left & $1 \mathrm{BKS}$ \\
\hline 28 & superior temporal & -52.1 & -17.8 & -4.4 & left & 1ST \\
\hline 29 & middle temporal & -55.6 & -31.1 & -12.9 & left & lMT \\
\hline 30 & inferior temporal & -48.9 & -34.4 & -22.2 & left & IIT \\
\hline 31 & temporal pole & -32.8 & 8.4 & -34.8 & left & ITP \\
\hline 32 & entorhinal & -25.8 & -7.6 & -31.6 & left & 1ENT \\
\hline 33 & parahippocampal & -24.7 & -31.2 & -17.4 & left & 1PHIP \\
\hline 34 & fusiform & -35.7 & -43.3 & -19.7 & left & 1FUS \\
\hline 35 & superior frontal & 13.4 & 24.7 & 42 & right & $\mathrm{rSF}$ \\
\hline 36 & frontal pole & 10.3 & 61.1 & -10 & right & $\mathrm{rFP}$ \\
\hline 37 & caudal anterior cingulate & 7.3 & 18.7 & 26.3 & right & $\mathrm{rCAC}$ \\
\hline 38 & caudal middle frontal & 34.9 & 11.8 & 43 & right & $\mathrm{rCMF}$ \\
\hline 39 & pars orbitalis & 42.1 & 39.2 & -10 & right & $\mathrm{rPOB}$ \\
\hline 40 & lateral orbitofrontal & 23.6 & 28.5 & -15.2 & right & rLOF \\
\hline 41 & pars triangularis & 45 & 29.7 & 4.5 & right & rPT \\
\hline 42 & pars opercularis & 44.9 & 14.4 & 14.2 & right & $\mathrm{rPOP}$ \\
\hline 43 & medial orbitofrontal & 8.8 & 35.7 & -14.8 & right & rMOF \\
\hline 44 & rostral middle frontal & 32.3 & 40.9 & 17.3 & right & rRMF \\
\hline 45 & rostral anterior cingulate & 8 & 33.5 & 2.1 & right & rRAC \\
\hline 46 & insula & 35.1 & -3.9 & 2.4 & right & rINS \\
\hline 47 & precentral & 36.8 & -9.9 & 43.5 & right & rPRC \\
\hline 48 & postcentral & 41.6 & -22.4 & 43.8 & right & rPOC \\
\hline 49 & supramarginal & 50.6 & -33.3 & 30.7 & right & rSUPRA \\
\hline 50 & superior parietal & 22.6 & -59.5 & 48.1 & right & rSP \\
\hline 51 & inferior parietal & 42.8 & -60.9 & 28.1 & right & rIP \\
\hline 52 & paracentral & 9.9 & -27.4 & 55.6 & right & rPARAC \\
\hline 53 & posterior cingulate & 7.6 & -17.1 & 36.2 & right & rPCG \\
\hline 54 & isthmus cingulate & 9.8 & -44.8 & 16.9 & right & rIST \\
\hline 55 & precuneus & 11.7 & -56.5 & 37.7 & right & rPREC \\
\hline 56 & cuneus & 8.7 & -80.1 & 19 & right & rCUN \\
\hline 57 & pericalcarine & 14 & -79.7 & 6.7 & right & rPERI \\
\hline
\end{tabular}


Table 2

(Continued)

\begin{tabular}{|c|c|c|c|c|c|c|}
\hline \multirow[t]{2}{*}{ No } & \multirow[t]{2}{*}{ Regions } & \multicolumn{3}{|c|}{$\begin{array}{l}\text { Montreal neurological } \\
\text { institute coordinates }\end{array}$} & \multirow[t]{2}{*}{$\mathrm{L} / \mathrm{R}$} & \multirow[t]{2}{*}{ label } \\
\hline & & $\mathrm{x}$ & $\mathrm{y}$ & $\mathrm{Z}$ & & \\
\hline 58 & lingual & 16.8 & -66.3 & -3.6 & right & $\mathrm{rLIN}$ \\
\hline 59 & lateral occipital & 30.3 & -86.3 & 0.5 & right & $\mathrm{rLO}$ \\
\hline 60 & transverse temporal & 44.8 & -22.4 & 6.5 & right & rTRANS \\
\hline 61 & banks superior temporal & 51.9 & -40.6 & 5.6 & right & $\mathrm{rBKS}$ \\
\hline 62 & superior temporal & 53 & -14 & -5.5 & right & $\mathrm{rST}$ \\
\hline 63 & middle temporal & 55.9 & -29.5 & -12.9 & right & rMT \\
\hline 64 & inferior temporal & 49.3 & -31.7 & -23 & right & rIT \\
\hline 65 & temporal pole & 34 & 8.4 & -33.1 & right & rTP \\
\hline 66 & entorhinal & 26.2 & -6.8 & -31.9 & right & rENT \\
\hline 67 & parahippocampal & 26.1 & -31.3 & -16.2 & right & rPHIP \\
\hline 68 & fusiform & 35.9 & -43 & -19.2 & right & rFUS \\
\hline
\end{tabular}

A non-parametric permutation test was performed to assess the global and nodal parameters in $A D$, DLB, and CN. A $p$-value of $<0.05$ after controlling for the family-wise error rate was considered significant with a two-tailed test of the null hypothesis.

\section{RESULTS}

The metabolic correlation matrix in $\mathrm{CN}$ was highly homogeneous in the whole brain; in contrast, the correlation matrices in AD and DLB were heterogeneous including lower correlation (Fig. 1). Some of the correlations decreased severely in DLB compared with $\mathrm{AD}$; however, other correlations in DLB were partially preserved. The 3D schematic figures clearly visualized the difference of the network topology between AD and DLB.

In the global parameters (Fig. 2), average strength, global efficiency, local efficiency, clustering coefficient, and transitivity were significantly lower in AD than in CN. Average eccentricity, average characteristic path length, and modularity were significantly higher in AD than in $\mathrm{CN}$. Similar results were obtained when DLB was compared with CN. Average strength, global efficiency, local efficiency, clustering coefficient, and transitivity were lower, and average eccentricity, average characteristic path length, and modularity were higher in DLB than in CN. However, no significant difference in the global parameters was found between AD and DLB.

In the nodal parameters (Fig. 3 and Table 3), significant differences in nodes were found between $\mathrm{AD}$ and DLB. The most remarkable node was the right posterior cingulate, which had lower strength, lower triangles, higher path length, lower global efficiency, lower clustering coefficient, and lower closeness centrality in DLB than in AD. The second most remarkable node was the left transverse temporal gyrus, which had a lower degree, higher path length, and lower closeness centrality in DLB than in AD.

\section{DISCUSSION}

We generated the metabolic connectivity using FDG-PET with the graph-theoretical method. The metabolic connections decreased in AD and DLB compared with $\mathrm{CN}$. The patterns of the decreased metabolic connections were different between AD and DLB, as shown by the difference in the nodal parameters of the specific nodes including the right posterior cingulate and the left transverse temporal gyrus.

In this study, patients clinically diagnosed with $\mathrm{AD}$ underwent $\left[{ }^{11} \mathrm{C}\right] \mathrm{PiB}-\mathrm{PET}$, and positive amyloid$\beta$ accumulation was confirmed. Recently, a new research framework has been published in NIA-AA [23]; however, we did not apply it in this study because we did not have enough data on tau protein for the studied population. DLB was diagnosed using the fourth consensus report (the newest version) of the DLB Consortium [20].

Functional connectivity analysis can be used to assess the integration of brain activity across distant brain regions. The graph-theoretical method can provide functional connectivity using time-series spatially parcellated data in functional MRI. Our idea is to input standardized patient-series data in FDG-PET to generate metabolic connectivity. The advantage of the graph-theoretical method combined with the standardized data is that we can analyze the whole brain data simultaneously without operators' controls. This analysis does not require predefined seeds or manual regions of interest. We can add 

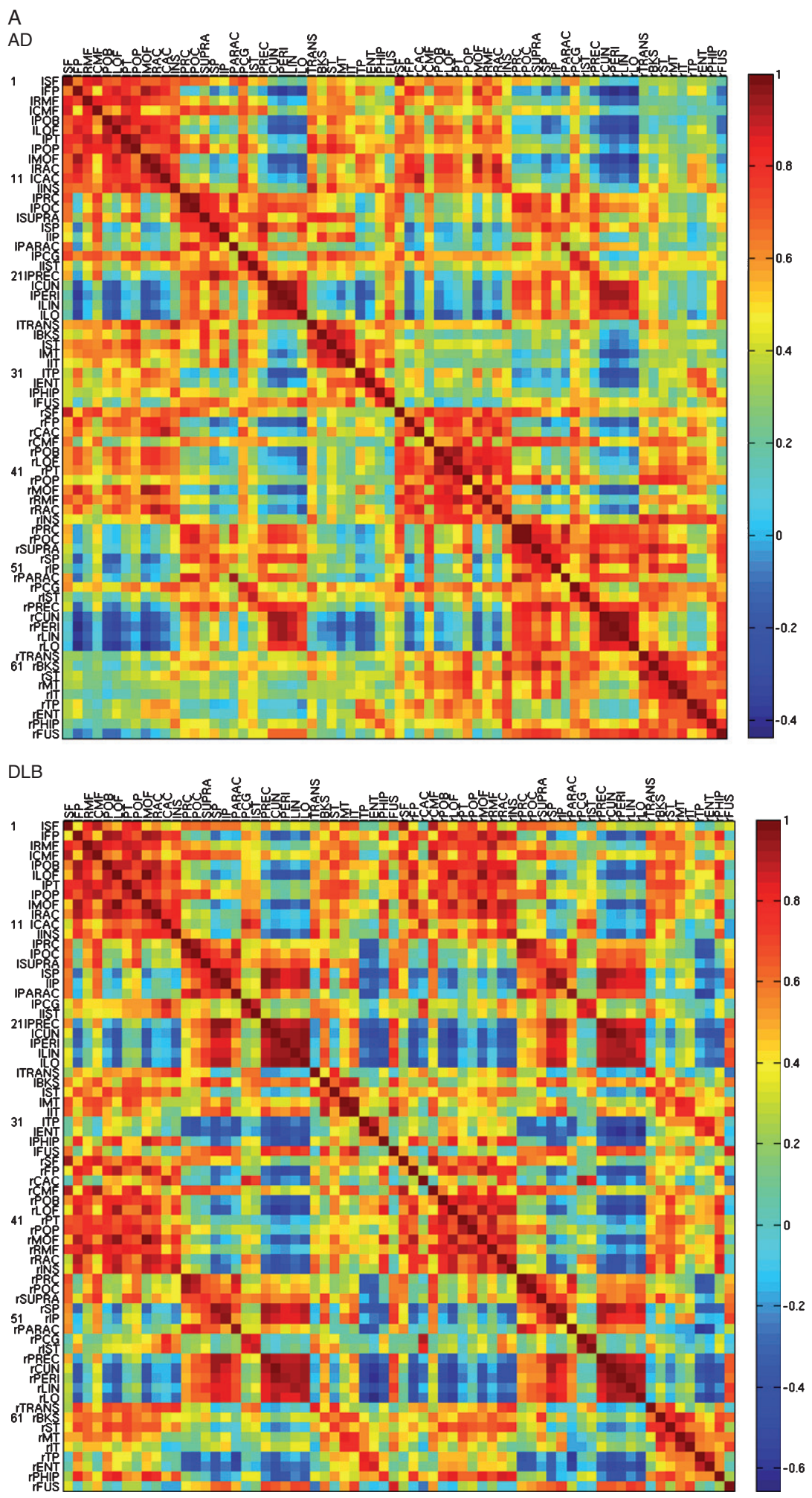

Fig. 1. (Continued) 

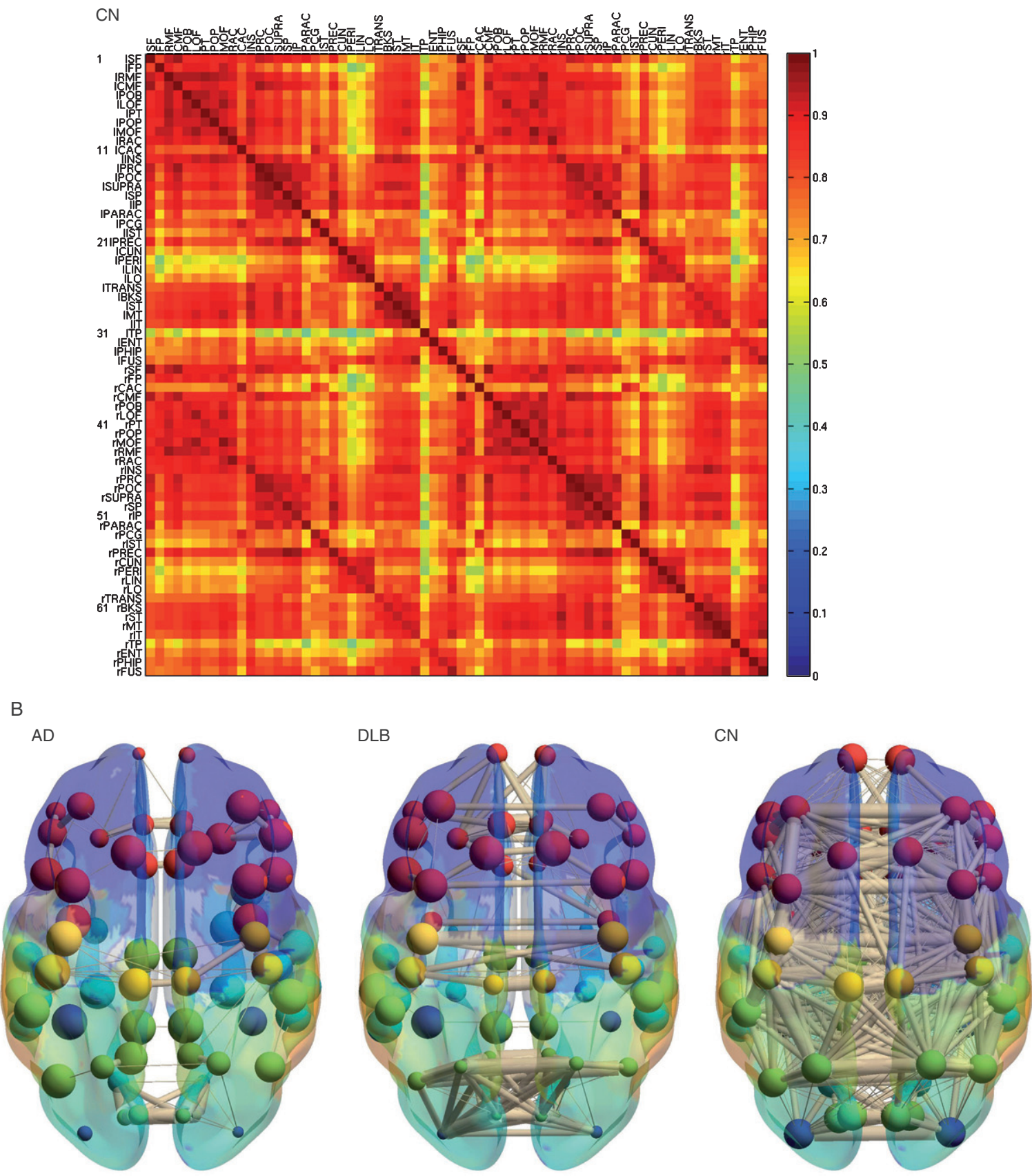

Fig. 1. Metabolic connectivity matrix and anatomical localizations. A) Metabolic connectivity matrix. The cell color in the correlation matrix indicates the magnitude of the correlation, and the color is arranged in gradation from red to blue in accordance with the magnitude of correlation from positive to negative. Note that the lower limit of the correlation range is different in each matrix (See a color navigation side bar). Some of the correlations decreased more severely in DLB than in AD. In addition, other correlations in DLB are relatively preserved. The texture of the matrix in DLB looks "patchy" compared with that in AD. AD, Alzheimer's disease; DLB, dementia with Lewy bodies; $\mathrm{CN}$, cognitive normal. B) The 3D schematic figures representing metabolic connectivity. The metabolic connections are overlaid on an anatomical atlas using nodes and edges. These figures are used to display an outline of the whole connectivity.

the noble information of metabolism over conventional FDG-PET image. The correlation matrix and 3D schematic figures clearly exhibited the dense net- work in $\mathrm{CN}$ and the sparse network in AD and DLB, and the global measures were significantly decreased in $\mathrm{AD}$ and $\mathrm{DLB}$ compared with $\mathrm{CN}$. Notably, the 

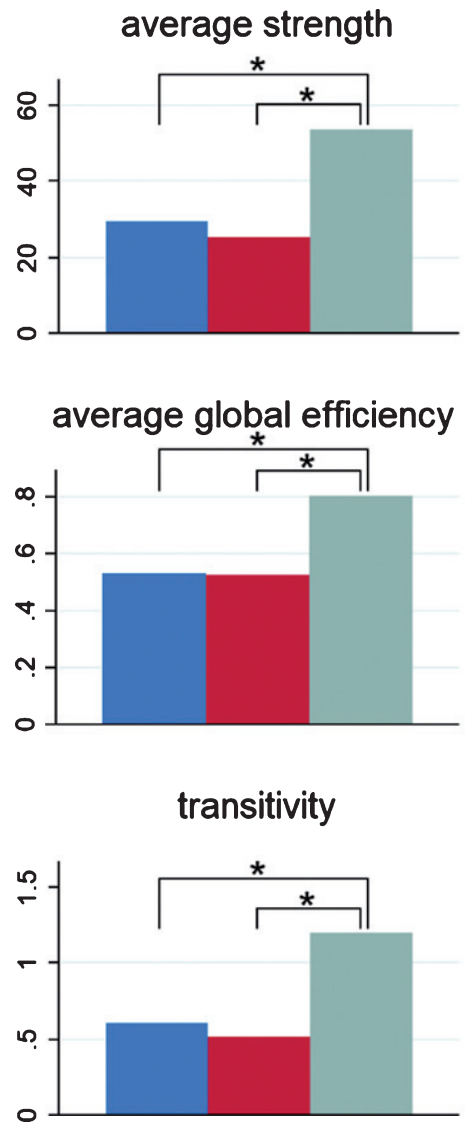

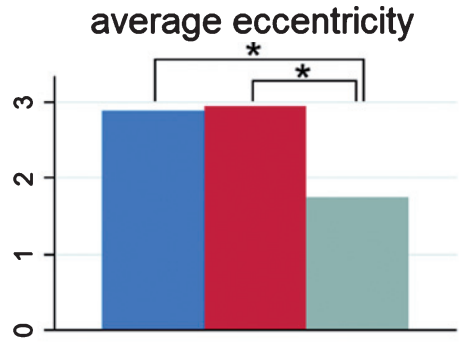

average characteristic path length
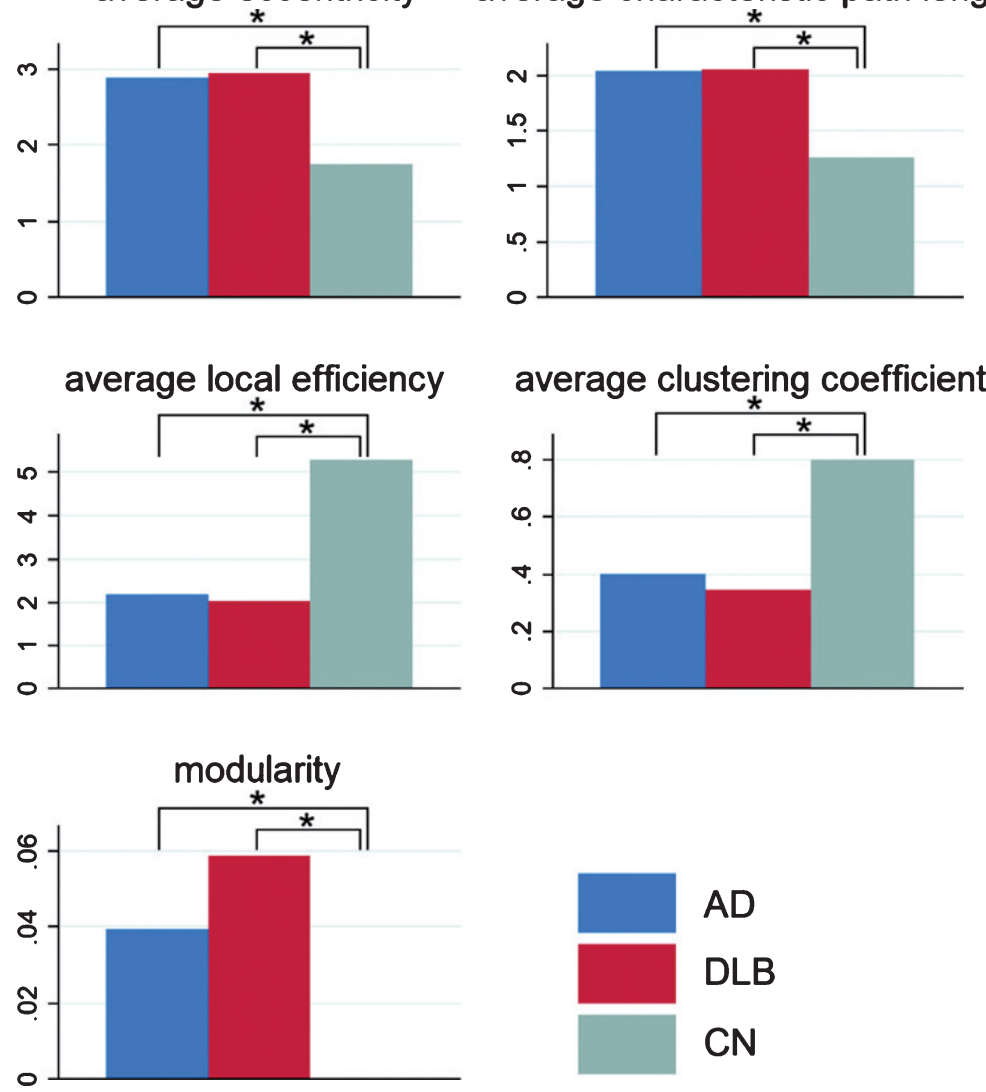

Fig. 2. Global parameters. Global parameters, including average strength, average eccentricity, average characteristic path length, average global efficiency, average local efficiency, average clustering coefficient, transitivity, and modularity, are displayed in the bar chart with blue bars for $\mathrm{AD}$, red bars for DLB, and green bars for CN. In all the parameters, significant differences were found in AD versus CN and DLB versus $\mathrm{CN}$, but no significant difference was found between AD and DLB. ${ }^{*} p<0.05$
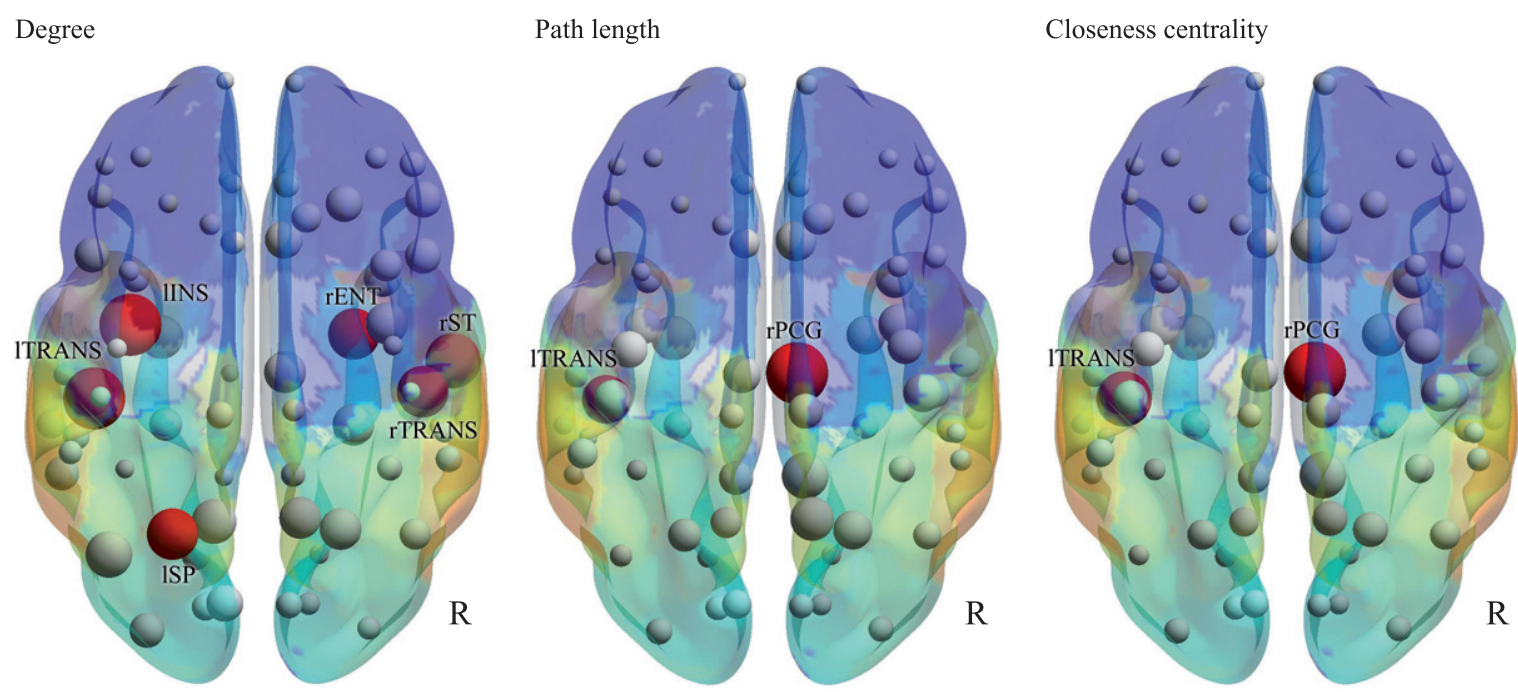

Fig. 3. Nodal parameters. Degree, path length, and closeness centrality are shown by 3D schematic figures. Red nodes indicate significant differences between AD and DLB, corresponding to Table 3. 
Table 3

Nodal parameters with significant differences between AD and DLB

\begin{tabular}{|c|c|c|c|c|c|}
\hline Brain regions & Measures & $\mathrm{AD}$ & DLB & Difference & $p$ \\
\hline right posterior cingulate & strength & 38.02 & 15.69 & -22.33 & $<0.01$ \\
\hline right posterior cingulate & triangles & 1049.5 & 372.9 & -676.6 & 0.03 \\
\hline right posterior cingulate & path length & 1.8538 & 3.2901 & 1.4363 & $<0.01$ \\
\hline right posterior cingulate & global efficiency of the nodes & 0.5688 & 0.3494 & -0.2194 & $<0.01$ \\
\hline right posterior cingulate & clustering nodes & 0.4747 & 0.2256 & -0.2491 & 0.02 \\
\hline right posterior cingulate & closeness centrality & 0.5394 & 0.3039 & -0.2355 & $<0.01$ \\
\hline left transverse temporal & degree & 67 & 50 & -17 & $<0.01$ \\
\hline left transverse temporal & path length & 2.0527 & 2.7779 & 0.7252 & 0.04 \\
\hline left transverse temporal & closeness centrality & 0.4872 & 0.3600 & -0.1272 & 0.02 \\
\hline left insula & degree & 67 & 51 & -16 & $<0.01$ \\
\hline left superior parietal & degree & 58 & 41 & -17 & $<0.01$ \\
\hline right transverse temporal & degree & 67 & 52 & -15 & $<0.01$ \\
\hline right superior temporal & degree & 67 & 52 & -15 & $<0.01$ \\
\hline right entorhinal & degree & 67 & 40 & -27 & $<0.01$ \\
\hline
\end{tabular}

global measures were not significantly different, but patterns of sparsity of the correlation matrices and the 3D schematic figures were different between AD and DLB. The horizontal connections, the commissures on either side, were relatively preserved in DLB compared with $\mathrm{AD}$; however, some of the connections were impaired more severely in DLB than in AD. These results suggest that DLB has restricted but deeper stereotyped network disruption than AD. We found several key nodes to differentiate DLB from $\mathrm{AD}$, including the right posterior cingulate and the left transverse temporal gyrus. The right posterior cingulate was relatively preserved compared with the left posterior cingulate in early AD. Cerebral blood flow is left-side dominantly decreased in $\mathrm{AD}$ [24, 25]. The left hemisphere is language-dominant and is related to the progression of clinical symptoms in AD. Moreover, in DLB, glucose metabolism is preserved in the posterior cingulate (cingulate island sign) [6]. Because the other cerebral cortex has decreased metabolism, the connection between the posterior cingulate and the other linked cortex decreases, possibly leading to significant differences in the metabolic connection in the right posterior cingulate. The transverse temporal gyrus is left-dominantly associated with auditory processing and has a wide network to the white matter. A previous pathological study revealed that the left transverse temporal gyrus was relatively spared for alpha-synuclein deposit [26]. Accordingly, the connections between the left transverse temporal gyrus and the other regions potentially decrease. Interestingly, both the right posterior cingulate and the left transverse temporal gyrus are involved in important nodes of DMN. In earlier studies of functional connectivity using functional MRI, it is controversial whether the DMN connection in DLB is different from that in $\mathrm{AD}$. Lowther et al. revealed that quite a few DMN connections were less in DLB than in AD [27]; however, Schumacher et al. found no decreased connection in DLB compared with AD [28]. Our study showed a part of DMN metabolic connections decreased more severely in DLB than in $\mathrm{AD}$, suggesting a partially decreased pattern in the correlation matrix in DLB.

The metabolic connectivity also revealed that the left insula, left superior parietal, right transverse temporal, right superior temporal, and right entorhinal nodes had lower degrees in DLB than in AD. The insula had significant volume loss in prodromal DLB compared with $\mathrm{CN}$ [29]. The volume of the insula was preserved in AD. Superior parietal and temporal regions are key regions for visuospatial activities and construction of visual perception [30, 31]. The parietal region is involved in the key connection that mediates retrieval of object representation from longterm memory through visual imagery [30]. Visual processing deficit is one of the specific symptoms in discriminating DLB [20]. DLB patients with typical visual hallucination show reduced FDG metabolism in the right occipitotemporal cortex [32]. The entorhinal cortex plays an important role not only in the storage and retrieval for the episodic memories [33] but also in the visuospatial recognition [34]. Actually, the dysfunction of the entorhinal cortex could result in impaired visual recognition in patients with DLB [35]. Atrophy of the entorhinal cortex is more severe in DLB than in AD [36]. An earlier study investigating hippocampal subfield atrophy in DLB revealed thinning of the right entorhinal cortex [37]. Moreover, visuospatial attention functions in the right hemisphere dominance [38]. These nodes are supplementary nodes for differentiating DLB from AD. 
This study has several limitations. A major limitation is a relatively small sample size, in particular in the DLB group. The small sample size might prevent us from detecting differences in topological parameters between DLB and AD, although the parameters could be significantly different. Second, the included patients in this study consisted of those diagnosed with AD or DLB only. Patients with combinationtype dementia were strictly excluded from this study. The combination-type dementia of AD and DLB could show mixed-characteristic patterns of the topological model.

\section{ACKNOWLEDGMENTS}

This study received no funding support, donation, or materials.

Authors' disclosures available online (https:// www.j-alz.com/manuscript-disclosures/19-0843r1).

\section{REFERENCES}

[1] Zaccai J, McCracken C, Brayne C (2005) A systematic review of prevalence and incidence studies of dementia with Lewy bodies. Age Ageing 34, 561-566.

[2] Sadiq D, Whitfield T, Lee L, Stevens T, Costafreda S, Walker Z (2017) Prodromal dementia with Lewy bodies and prodromal Alzheimer's disease: A comparison of the cognitive and clinical profiles. J Alzheimers Dis 58, 463-470.

[3] Tousi B (2017) Diagnosis and management of cognitive and behavioral changes in dementia with Lewy bodies. Curr Treat Options Neurol 19, 42.

[4] Sanford AM (2018) Lewy body dementia. Clin Geriatr Med 34, 603-615.

[5] Minoshima S, Foster NL, Sima AA, Frey KA, Albin RL, Kuhl DE (2001) Alzheimer's disease versus dementia with Lewy bodies: Cerebral metabolic distinction with autopsy confirmation. Ann Neurol 50, 358-365.

[6] Lim SM, Katsifis A, Villemagne VL, Best R, Jones G, Saling M, Bradshaw J, Merory J, Woodward M, Hopwood M, Rowe CC (2009) The 18F-FDG PET cingulate island sign and comparison to 123I-beta-CIT SPECT for diagnosis of dementia with Lewy bodies. J Nucl Med 50, 1638-1645.

[7] Mosconi L, Tsui WH, Herholz K, Pupi A, Drzezga A, Lucignani G, Reiman EM, Holthoff V, Kalbe E, Sorbi S, Diehl-Schmid J, Perneczky R, Clerici F, Caselli R, Beuthien-Baumann B, Kurz A, Minoshima S, de Leon MJ (2008) Multicenter standardized 18F-FDG PET diagnosis of mild cognitive impairment, Alzheimer's disease, and other dementias. J Nucl Med 49, 390-398.

[8] Biswal B, Yetkin FZ, Haughton VM, Hyde JS (1995) Functional connectivity in the motor cortex of resting human brain using echo-planar MRI. Magn Reson Med 34, 537541.

[9] Roy CS, Sherrington CS (1890) On the regulation of the blood-supply of the brain. J Physiol 11, 85-158 117.

[10] Attwell D, Laughlin SB (2001) An energy budget for signaling in the grey matter of the brain. J Cereb Blood Flow Metab 21, 1133-1145.
[11] Baron JC, Lebrun-Grandie P, Collard P, Crouzel C, Mestelan G, Bousser MG (1982) Noninvasive measurement of blood flow, oxygen consumption, and glucose utilization in the same brain regions in man by positron emission tomography: Concise communication. J Nucl Med 23, 391-399.

[12] Raichle ME, MacLeod AM, Snyder AZ, Powers WJ, Gusnard DA, Shulman GL (2001) A default mode of brain function. Proc Natl Acad Sci U S A 98, 676-682.

[13] Greicius MD, Krasnow B, Reiss AL, Menon V (2003) Functional connectivity in the resting brain: A network analysis of the default mode hypothesis. Proc Natl Acad Sci U S A 100, 253-258.

[14] Greicius MD, Srivastava G, Reiss AL, Menon V (2004) Default-mode network activity distinguishes Alzheimer's disease from healthy aging: Evidence from functional MRI. Proc Natl Acad Sci U S A 101, 4637-4642.

[15] Binnewijzend MA, Schoonheim MM, Sanz-Arigita E, Wink AM, van der Flier WM, Tolboom N, Adriaanse SM, Damoiseaux JS, Scheltens P, van Berckel BN, Barkhof F (2012) Resting-state fMRI changes in Alzheimer's disease and mild cognitive impairment. Neurobiol Aging 33, 2018-2028.

[16] Petrella JR, Sheldon FC, Prince SE, Calhoun VD, Doraiswamy PM (2011) Default mode network connectivity in stable vs progressive mild cognitive impairment. Neurology 76, 511-517.

[17] Galvin JE, Price JL, Yan Z, Morris JC, Sheline YI (2011) Resting bold fMRI differentiates dementia with Lewy bodies vs Alzheimer disease. Neurology 76, 1797-1803.

[18] Kenny ER, Blamire AM, Firbank MJ, O'Brien JT (2012) Functional connectivity in cortical regions in dementia with Lewy bodies and Alzheimer's disease. Brain 135, 569-581.

[19] McKhann GM, Knopman DS, Chertkow H, Hyman BT, Jack CR, Jr., Kawas CH, Klunk WE, Koroshetz WJ, Manly JJ, Mayeux R, Mohs RC, Morris JC, Rossor MN, Scheltens P, Carrillo MC, Thies B, Weintraub S, Phelps $\mathrm{CH}$ (2011) The diagnosis of dementia due to Alzheimer's disease: Recommendations from the National Institute on Aging-Alzheimer's Association workgroups on diagnostic guidelines for Alzheimer's disease. Alzheimers Dement 7, 263-269.

[20] McKeith IG, Boeve BF, Dickson DW, Halliday G, Taylor JP, Weintraub D, Aarsland D, Galvin J, Attems J, Ballard CG, Bayston A, Beach TG, Blanc F, Bohnen N, Bonanni L, Bras J, Brundin P, Burn D, Chen-Plotkin A, Duda JE, ElAgnaf O, Feldman H, Ferman TJ, Ffytche D, Fujishiro H, Galasko D, Goldman JG, Gomperts SN, Graff-Radford NR, Honig LS, Iranzo A, Kantarci K, Kaufer D, Kukull W, Lee VMY, Leverenz JB, Lewis S, Lippa C, Lunde A, Masellis M, Masliah E, McLean P, Mollenhauer B, Montine TJ, Moreno E, Mori E, Murray M, O’Brien JT, Orimo S, Postuma RB, Ramaswamy S, Ross OA, Salmon DP, Singleton A, Taylor A, Thomas A, Tiraboschi P, Toledo JB, Trojanowski JQ, Tsuang D, Walker Z, Yamada M, Kosaka K (2017) Diagnosis and management of dementia with Lewy bodies: Fourth consensus report of the DLB Consortium. Neurology 89, 88-100.

[21] Mijalkov M, Kakaei E, Pereira JB, Westman E, Volpe G (2017) BRAPH: A graph theory software for the analysis of brain connectivity. PLoS One 12, e0178798.

[22] Xia M, Wang J, He Y (2013) BrainNet Viewer: A network visualization tool for human brain connectomics. PLoS One 8, e68910.

[23] Jack CR, Jr., Bennett DA, Blennow K, Carrillo MC, Dunn B, Haeberlein SB, Holtzman DM, Jagust W, Jessen F, Karlawish J, Liu E, Molinuevo JL, Montine T, Phelps C, Rankin 
KP, Rowe CC, Scheltens P, Siemers E, Snyder HM, Sperling R (2018) NIA-AA Research Framework: Toward a biological definition of Alzheimer's disease. Alzheimers Dement 14, 535-562.

[24] Ishii K, Sasaki M, Yamaji S, Sakamoto S, Kitagaki H, Mori E (1997) Demonstration of decreased posterior cingulate perfusion in mild Alzheimer's disease by means of $\mathrm{H} 215 \mathrm{O}$ positron emission tomography. Eur J Nucl Med 24, 670-673.

[25] Huang C, Wahlund LO, Svensson L, Winblad B, Julin P (2002) Cingulate cortex hypoperfusion predicts Alzheimer's disease in mild cognitive impairment. $B M C$ Neurol 2, 9.

[26] Yamamoto R, Iseki E, Murayama N, Minegishi M, Marui W, Togo T, Katsuse O, Kosaka K, Kato M, Iwatsubo T, Arai H (2007) Correlation in Lewy pathology between the claustrum and visual areas in brains of dementia with Lewy bodies. Neurosci Lett 415, 219-224.

[27] Lowther ER, O'Brien JT, Firbank MJ, Blamire AM (2014) Lewy body compared with Alzheimer dementia is associated with decreased functional connectivity in resting state networks. Psychiatry Res 223, 192-201.

[28] Schumacher J, Peraza LR, Firbank M, Thomas AJ, Kaiser M, Gallagher P, O’Brien JT, Blamire AM, Taylor JP (2018) Functional connectivity in dementia with Lewy bodies: A within- and between-network analysis. Hum Brain Mapp 39, 1118-1129.

[29] Blanc F, Colloby SJ, Cretin B, de Sousa PL, Demuynck C, O'Brien JT, Martin-Hunyadi C, McKeith I, Philippi N, Taylor JP (2016) Grey matter atrophy in prodromal stage of dementia with Lewy bodies and Alzheimer's disease. Alzheimers Res Ther $\mathbf{8}, 31$.

[30] Ishai A, Ungerleider LG, Haxby JV (2000) Distributed neural systems for the generation of visual images. Neuron 28, 979-990.
[31] Mechelli A, Price CJ, Friston KJ, Ishai A (2004) Where bottom-up meets top-down: Neuronal interactions during perception and imagery. Cereb Cortex 14, 1256-1265.

[32] Iaccarino L, Sala A, Caminiti SP, Santangelo R, Iannaccone S, Magnani G, Perani D (2018) The brain metabolic signature of visual hallucinations in dementia with Lewy bodies. Cortex 108, 13-24.

[33] Di Paola M, Macaluso E, Carlesimo GA, Tomaiuolo F, Worsley KJ, Fadda L, Caltagirone C (2007) Episodic memory impairment in patients with Alzheimer's disease is correlated with entorhinal cortex atrophy. A voxel-based morphometry study. J Neurol 254, 774-781.

[34] Connor CE, Knierim JJ (2017) Integration of objects and space in perception and memory. Nat Neurosci 20, 14931503.

[35] Mondon K, Gochard A, Marque A, Armand A, Beauchamp D, Prunier C, Jacobi D, de Toffol B, Autret A, Camus V, Hommet C (2007) Visual recognition memory differentiates dementia with Lewy bodies and Parkinson's disease dementia. J Neurol Neurosurg Psychiatry 78, 738-741.

[36] Kenny ER, Burton EJ, O'Brien JT (2008) A volumetric magnetic resonance imaging study of entorhinal cortex volume in dementia with lewy bodies. A comparison with Alzheimer's disease and Parkinson's disease with and without dementia. Dement Geriatr Cogn Disord 26, 218-225.

[37] Delli Pizzi S, Franciotti R, Bubbico G, Thomas A, Onofrj M, Bonanni L (2016) Atrophy of hippocampal subfields and adjacent extrahippocampal structures in dementia with Lewy bodies and Alzheimer's disease. Neurobiol Aging 40, 103-109.

[38] Thiebaut de Schotten M, Dell'Acqua F, Forkel SJ, Simmons A, Vergani F, Murphy DG, Catani M (2011) A lateralized brain network for visuospatial attention. Nat Neurosci 14, 1245-1246. 